Revista de Psicología de la PUCP. Vol. XXII, 1, 2004

\title{
La evaluación de los estilos de liderazgo en población civil y militar argentina ${ }^{1}$
}

\author{
Alejandro Castro Solano ${ }^{2}$, Martín Nader ${ }^{3}$ \\ Colegio Militar de la Nación, Argentina
}

\author{
María Martina Casullo ${ }^{4}$ \\ Universidad de Buenos Aires \\ Consejo Nacional de Investigaciones \\ Científicas y Técnicas, Argentina
}

Basados en la teoría de Bass (1985, 1990), se adaptó el cuestionario M.L.Q. (Multifactor Leadership Questionnaire) para la evaluación de los estilos de liderazgo civiles y militares argentinos. Se recogieron datos de 363 sujetos, civiles (53\%) y militares cadetes y oficiales ( $47 \%$ ). Se realizó un análisis factorial exploratorio y luego confirmatorio resultando una escala de 34 ítems que responde a un modelo de siete factores agrupados en tres estilos de liderazgo. Los resultados señalan mejor ajuste del modelo para población civil que para militar. Se encontraron diferencias individuales entre los estilos de liderazgo de civiles y militares. En los primeros predominan las características relacionadas con el intercambio y la negociación. En los ámbitos militares predomina el estilo de liderazgo transformacional. En ambas poblaciones las características más valoradas de los líderes se autopercibían con mayor intensidad en el formato de autoregistro que en la percepción del subalterno respecto del superior.

Palabras clave: liderazgo, población militar, población civil, MLQ.

\section{Assessment of leadership styles of military and civil argentines}

Based on Bass' leadership model (1985, 1990), the M.L.Q. questionnaire (Multifactor Leadership Questionnaire) was validated for the assessment of leadership styles in Argentinean civil and military population. Data were gathered $(\mathrm{N}=363)$ on military (cadets and officers) and civilian population. A factorial structure of seven grouped in three styles of leadership (34 items) was obtained and it was confirmed by means of confirmatory factor analysis. The results point out better adjustment of the obtained pattern for civil population than for military. Individual differences were also found among the styles of leadership of civilians and military population. In the first ones characteristics related with the exchange and the negotiation prevail. In the military environments The style of transformational leadership prevails in military environments. In both populations valued leader characteristics were perceived with more intensity in self-perception format than in the perception of subordinates regarding their superiors.

Key words: leadership, military, civilians, MLQ.

Proyecto subsidiado por la Secretaría de Ciencia y Tecnología de la Nación. SECYT 0242/03. cias profesionales en ámbitos militares", Ejército Argentino. Ministerio de Defensa. Correo electrónico: alito@fibertel.com.ar 

El estudio del liderazgo y la capacidad de conducción en los grupos de trabajo ha sido una de las variables a las que la investigación sobre psicología de las organizaciones ha prestado mayor importancia (Morales \& Molero, 1995). La mayoría de las definiciones del liderazgo consideran que es un proceso eminentemente psicosocial, en el cual una persona ejerce una influencia intencional sobre un grupo con el propósito de guiar, estructurar o facilitar una tarea en función de un objetivo colectivo (Yukl, 2002). Las mayores discrepancias en la literatura se localizan en quién y cómo ejerce esa influencia, el beneficiario de la misma (seguidor) y el resultado del proceso (efectividad).

El paradigma dominante en el estudio del liderazgo evolucionó desde modelos más estáticos basados en los rasgos psicológicos de los líderes a modelos situacionales que tomaban en cuenta las variables del ambiente y de la persona (Yukl, 2002). El grupo de Ohio, EE.UU., dominó las investigaciones sobre el liderazgo desde los años 50 y hasta entrados los 80 . Según esta línea de investigación, el líder tenía que ejercer dos conductas básicas: consideración (importancia que el líder otorga a los miembros de su grupo) e iniciación de estructura (capacidad para llevar a cabo metas, coordinar actividades y mantener el control del grupo). El presupuesto básico de este modelo señala que el liderazgo consiste en una serie de transacciones o intercambios entre líder y seguidor. Si las conductas ejercidas son efectivas debe observarse un correlato con otros constructos tales

Licenciado en Psicología. Becario del Proyecto.

Doctora en Psicología. Profesora en la Facultad de Psicología, Universidad de Buenos Aires. Coordinadora del Proyecto como Investigadora del CONICET. 
como productividad y satisfacción en el trabajo (Stogdill, Good \& Day, 1962).

En la década del 80 surgieron aproximaciones teóricas alternativas. La principal crítica (Trice \& Beyer, 1993) radicó en que se hacía referencia sólo al liderazgo de bajo nivel, considerando solo los aspectos instrumentales del mismo (intercambios diarios entre líder/ seguidor). Los resultados de las investigaciones que utilizaron como predictores la iniciación de estructura y la consideración han sido inconsistentes y débiles (Bass, 1990; Fisher \& Edwards, 1988). Muchos de los aspectos que influyen en el liderazgo son contextuales y derivados de la cultura organizacional. Estos aspectos son frecuentemente implícitos, así como la propia concepción del líder que tienen las personas en función de la cual se acepta o se rechaza la conducta del mismo (House, Nages, Ruiz Quintanilla \& Dorfman, 1999). Tanto los líderes como los seguidores poseen un guión o estereotipo sobre cuáles son las conductas esperadas de una persona para ser considerada líder, variables eminentemente contextuales (Wofford, Godwin \& Wittington, 1998). Las investigaciones demostraron que las personas utilizan este proceso atribucional para categorizar a los líderes (Cantor \& Mischel, 1979).

Burns (1978) propuso un tipo de liderazgo transformacional en el cual el líder insta al grupo a trascender sus intereses personales en función de una visión de futuro. El líder tiene que proveer cuidado a sus seguidores y éstos a cambio prometen seguirlo para desarrollar la visión. Bass (1985), sobre la base de estas ideas, desarrolló una teoría sobre el liderazgo transformacional.

Bass (1985) afirma que las teorías del liderazgo se focalizaron exclusivamente en los intercambios que se producían entre el líder y los seguidores, en la clarificación de objetivos y metas y la sanción derivada según se alcancen o no las mismas. El autor afirma que una teoría del liderazgo debe explicar cómo los seguidores trascienden 
sus propios intereses personales por los objetivos del grupo o de la institución para alcanzar niveles óptimos de rendimiento. El líder (llamado en este caso transformacional o carismático) actúa sintetizando la información del medio, donde su propio sistema de valores se vuelve un organizador importante para los seguidores. El foco de esta nueva teoría del liderazgo se basa en los componentes transformacionales de los líderes para elevar la motivación y el compromiso de los seguidores. En concreto, este estilo de liderazgo transforma el autoconcepto de los seguidores (Shamir, House \& Arthur, 1993), identificando las metas personales de la organización y suplantando el sistema de valores personales por los del líder. El compromiso logrado por los líderes transformacionales provee cohesión y permite a los grupos enfrentar dificultades o retos importantes.

Hunt (1991) afirma que hubo un resurgimiento del estudio del liderazgo a mediados de los 80 y durante los 90 en los ámbitos educativos y organizacionales. Las investigaciones en los últimos 10 años estuvieron focalizadas en los efectos del liderazgo transformacional, especialmente en los aspectos motivacionales de los seguidores y en la productividad derivada de la efectividad del mismo (Avolio, 1999; Bass \& Avolio, 1994, 1997; Conger \& Kanungo, 1988). Según Bass, Avolio, Jung y Berson (2003) el liderazgo transformacional está positivamente correlacionado con la evaluación de los supervisores en ámbitos organizacionales, las recomendaciones para promoción de los empleados, las innovaciones en los equipos de trabajo y las metas financieras que alcanzan las unidades de negocios en las empresas. Estos hallazgos fueron confirmados por estudios metaanalíticos realizados por Lowe, Kroek y Sivasubramaniam (1996) y por Paterson, Fuller, Kester y Stringer (1995). Según Antonakis, Avolio y Sivasubramaniam (2003) los estudios sobre el liderazgo se hallan en la fase dos, en la cual las teorías son revisadas críticamente por los autores y el foco de los estudios consiste en la revisión de las variables moderadoras (básicamente contextuales) relevantes a las teorías. 
El instrumento derivado de la teoría de Bass para la evaluación de los estilos de liderazgo ha sido el cuestionario MLQ (Multifactor Leadership Questionnaire) que ha sufrido extensas revisiones (Avolio, Bass \& Jung, 1995), en parte debido a las dimensiones que componían cada uno de los estilos básicos del liderazgo (transformacional y transaccional). Antonakis et al. (2003) comentan que se han empleado metodologías diferentes para el análisis de las propiedades psicométricas del MLQ en cada uno de los estudios consultados. Si bien todas las versiones exhiben alta consistencia interna, la solución factorial alcanzada varía de un estudio a otro (de un modelo de tres a un modelo de nueve factores). Las primeras versiones confirman la estructura original propuesta por Bass de cinco o seis factores del liderazgo. Un problema del MLQ consiste en que algunas dimensiones del liderazgo no tenían la validez discriminante suficiente (por ejemplo, carisma/inspiración motivacional, laissez faire y manejo por la excepción, que saturaban en un mismo factor). Otra crítica se halla en relación con la multicolinearidad de las escalas que componen cada estilo de liderazgo. La alta correlación entre las dimensiones del liderazgo para cada escala hace sospechar que se trate realmente de dimensiones diferentes (Bycio, Hackett \& Allen, 1995; Carless, 1998). Muchos de los estudios comentados emplearon técnicas de análisis factorial exploratorio, lo cual no es un procedimiento adecuado para arribar a la validez de constructo de un instrumento, debiendo emplearse técnicas de análisis factorial confirmatorio (Antonakis et al., 2003). Asimismo, muchos estudios resultan incomparables en algunos casos ya que existen seis versiones diferentes del mismo instrumento. En la actualidad la versión más avanzada es el MLQ forma x 5 (Avolio et al., 1995). Para este estudio se trabajó con una versión española anterior y adaptada previamente al castellano por Morales y Molero (1995).

En virtud de la revisión bibliográfica realizada se diseñó una investigación que tiene como objetivo general validar el cuestionario MLQ (versión española) en dos tipos de poblaciones: civil y militar. 
El estudio que se presenta es parte de una investigación más amplia que tiene como propósito verificar la efectividad de un programa de entrenamiento de jóvenes líderes (cadetes). La dimensión de liderazgo transformacional se considera esencial para el ámbito militar ya que la moral, la cohesión y el compromiso generado por este estilo de liderazgo son los ingredientes esenciales del nivel de efectividad de una unidad (Bass, 1998; Gal, 1985). En términos generales la bibliografía señala que un alto espíritu de cuerpo y moral en una unidad militar produce los mejores resultados (Shamir, Zakay, Breinin \& Popper, 1998). Se espera que los líderes transformacionales eleven la efectividad de sus unidades fijando altas expectativas de logro y generando mayor compromiso, especialmente cuando surgen obstáculos y dificultades (Avolio, 1999; Bass, 1998). Yekeay (2002) afirma que los líderes militares son diferentes de los líderes en otros tipos de organizaciones ya que éstos son asignados a sus cargos y no emergen de forma natural. El autor comenta que los líderes militares tienen dos funciones muy delimitadas: las de ser un especialista en la tarea y en la conducción de personal, especialmente en mantener la cohesión en situaciones de alto estrés. En función de lo comentado, el estudio tiene los siguientes objetivos:

- Validar el cuestionario MLQ, estableciendo las propiedades psicométricas básicas para población local utilizando técnicas de análisis factorial confirmatorio, reduciendo los ítems del inventario para tener una prueba validada de un número bajo de ítems.

- Analizar diferencias individuales en los estilos de liderazgo predominante en población civil y militar (cadetes y oficiales).

- Verificar si existen diferencias entre la percepción del líder (superior) y la propia autopercepción de liderazgo en las muestras estudiadas. 


\section{Metodología}

\section{Participantes}

Participaron del estudio 363 sujetos, civiles y militares. El 47\% de los participantes eran militares (cadetes, $n=111$ y oficiales, $n=61$ ). El 53\% restante era población general no consultante $(n=191)$. Los cadetes eran jóvenes estudiantes en una institución militar perteneciente a las Fuerzas Armadas Argentinas. Estos jóvenes realizan un programa de entrenamiento combinado académico/militar de 4 años de duración. Se consideraron cadetes en los últimos años de su carrera y próximos a su graduación (3er. y 4to. año). Se trata en su mayoría de varones con un promedio de edad de 22,01 $(D E=1.90)$. El resto de la población militar son oficiales en los primeros tramos de su carrera militar. Tenían en promedio $26,85(D E=6.69)$. La población general $(n=191)$ era adulta, varones $(n=108,56 \%)$ y mujeres $(n=83,44 \%)$, que tenían en promedio 39,18 años (Desv. Típica $=$ 11.43). Eran personas que tenían algún puesto de dirección y poseían personal a cargo. El $40 \%(n=62)$ pertenecía a empresas pequeñas, el $35 \%$ se desempeñaba en empresas medianas $(n=55)$ y el restante $25 \%$ se desempeñaba en grandes empresas $(n=39)$. De acuerdo al cargo desempeñado podemos agrupar a los participantes en tres grupos definidos de mayor a menor categoría ocupacional. El $46 \%$ eran funcionarios, directivos y/o profesionales (se trataba de la categoría ocupacional más alta, $n=77$ ). El $27 \%$ eran jefes de nivel intermedio, supervisores y bancarios calificados $(n=46)$. El $27 \%$ restante $(n=45)$ se trataba de comerciantes, cuentapropistas y docentes. Todos los participantes civiles y militares residían en la ciudad de Buenos Aires y el conurbano bonaerense.

\section{Instrumentos}

a) Cuestionario MLQ (Multifactor Leadership Questionnaire) (Bass \& Avolio, 1990): es un cuestionario de 70 ítems que operacionaliza 
la teoría del liderazgo transformacional/transaccional de los autores. Trabajamos con la versión española de Morales y Molero (1995). Se realizó una adaptación de la misma para población argentina, reduciéndose la cantidad de ítems, ya que se necesitaba contar con un instrumento breve para realizar seguimientos a mediano plazo de los participantes. La escala final posee 34 ítems. Los participantes respondieron la forma autoadministrable. Una forma similar fue contestada por los subordinados respecto de las características del superior. La prueba presenta fiabilidad y validez adecuadas (ver Resultados). Las dimensiones que componen el instrumento son:

- Liderazgo Transformacional. Tiene cuatro subdimensiones: a) Carisma ó Influencia idealizada, respeto por el líder que quiere ser imitado y que formula altos niveles de expectativa en sus seguidores; b) Inspiración, es el grado en que el líder energiza a sus seguidores proveyendo una visión de futuro, objetivos y propósito; c) Estimulación intelectual, señala las acciones del líder relacionada con el estímulo intelectual para que los seguidores resuelvan los problemas de forma creativa, nueva ante dificultades y obstáculos; d) Consideración individualizada, es el apoyo que se provee al seguidor, prestando importancia a sus necesidades de desarrollo personal.

- Liderazgo Transaccional. Tiene dos subdimensiones: a) Recompensa contingente, es una interacción entre líder y seguidor guiada por intercambios recíprocos. El líder identifica las necesidades de los seguidores y realiza una transacción entre las necesidades del grupo y las de cada persona. Recompensa ó sanciona en función del cumplimiento de objetivos; b) Manejo por excepción, el líder interviene solo cuando hay que hacer correcciones o cambios en las conductas de los seguidores. En general las intervenciones son negativas y de crítica para que los objetivos no se desvíen de su curso.

- Laissez Faire. Representa la ausencia de transacción de cualquier clase. El líder evita tomar decisiones, no tiene responsabilidad y 
no usa su autoridad. Es considerada la forma más inefectiva de liderazgo.

b) Calificaciones académicas: el rendimiento militar fue tomado de los registros computarizados derivado del promedio de calificaciones que obtuvieron los cadetes durante el segundo semestre de 2002. Se utilizó una puntuación agregada de los diferentes aspectos que evalúan el rendimiento militar.

c) Escala de satisfacción: se diseñó una escala ad-hoc que evaluaba el grado de satisfacción en el trabajo para población civil. Tenía cuatro ítems que evaluaban diferentes aspectos de la satisfacción laboral: a) del trabajo en general; b) con el superior; c) con los compañeros de trabajo y d) con el clima de trabajo. Tenía formato Likert con siete opciones de respuesta. Se utilizó una puntuación agregada de los cuatro ítems.

\section{Procedimiento}

En cuanto a la población militar la participación tanto de estudiantes como oficiales fue voluntaria. Se administraron los protocolos de forma colectiva a los estudiantes y de forma individual a los oficiales. Las pruebas administradas formaban parte de una batería más amplia sobre valores y liderazgo. En cuanto a la población civil se localizaron personas que tuvieran algún puesto de dirección en empresas. Un grupo de estudiantes de psicología que estaban realizando sus prácticas de investigación recolectó las pruebas. Cada estudiante recogió 10 protocolos al azar en población general, obteniéndose el fondo de protocolos consignado en la descripción de la muestra. Los protocolos con omisiones fueron descartados y no se consignaron en el estudio. Un psicólogo graduado coordinó las tareas de administración y la tabulación de los datos cualitativos. Las tareas de data entry fueron realizadas por personal administrativo de la institución donde se llevó a cabo el estudio. 


\section{Resultados}

\section{Validez de constructo del MLQ-AVA}

\section{Análisis factorial exploratorio}

Se analizó la estructura factorial del MLQ. Se partió de la versión española del MLQ que contaba con 70 ítems. Como primer paso se analizó la correlación entre cada ítem y la escala para la que había sido originalmente diseñado con el propósito de homogeneizar el contenido de las mismas y conservar los elementos que dieran por resultado una mejor fiabilidad, por lo tanto, los ítems con correlaciones ítem-escala bajas $(r<0.30)$ fueron eliminados. Luego se llevó a cabo un análisis factorial de componentes principales con rotación Varimax de sus elementos. El proceso fue llevado a cabo de forma iterativa eliminándose en cada paso los ítems que tuvieran peso similar en más de un factor. La solución factorial final (Test de esfericidad de Bartlet $=3601,40 p<0.01$; Índice Káiser Meyer Olkin $=0.85$ ) constaba de siete factores que explicaban el $52.25 \%$ de la varianza de las puntuaciones. Los factores resultantes coinciden con las dimensiones teóricas propuestas por los autores de la prueba (Ver Cuadro 1).

\section{Cuadro 1}

Estructura factorial del $M L Q-A V A$

\begin{tabular}{|c|c|c|c|c|c|c|}
\hline Componentes & Factor 1 Factor & Factor & Factor & Factor & actor & actor 7 \\
\hline Varianza explicada & 18.16 & 6.39 & 4.78 & 3.97 & 3.56 & 3.24 \\
\hline $\begin{array}{l}\text { Les hago pensar sobre } \\
\text { viejos problemas de } \\
\text { forma nueva }\end{array}$ & 0.69 & & & & & \\
\hline $\begin{array}{l}\text { Les pido que fundamenten } \\
\text { sus opiniones con } \\
\text { argumentos sólidos }\end{array}$ & 0.69 & & & & & \\
\hline $\begin{array}{l}\text { Pongo especial énfasis } \\
\text { en la solución cuidadosa } \\
\text { de los problemas antes de } \\
\text { actuar }\end{array}$ & 0.62 & & & & & \\
\hline
\end{tabular}


Hago que se basen en el razonamiento y en la evidencia para resolver problemas

Impulso la utilización de la inteligencia para superar obstáculos

Trato de que vean los problemas como una oportunidad para aprender

Les doy nuevas formas de enfocar los problemas que antes resultaban desconcertantes

Es probable que esté ausente cuando se me necesita

Soy difícil de encontrar cuando surge un problema

Evito tomar decisiones

No les digo dónde me sitúo en algunas ocasiones

Evito decirles cómo se tienen que hacer las cosas

Mi presencia tiene poco efecto con su rendimiento

No trato de cambiar lo que hacen mientras las cosas salgan bien

No trato de hacer cambios mientras las cosas marchen bien

Les dejo que sigan haciendo su trabajo como siempre lo han hecho, si no me parece necesario introducir algún cambio

Evito intervenir, excepto cuando no se consiguen los objetivos 
La evaluación de los estilos de liderazgo en población civil y militar argentina

Componentes

Factor 1 Factor 2 Factor 3Factor 4 Factor 5 Factor 6 Factor 7

Demuestro que creo

firmemente en el dicho

"si funciona, no lo arregles"

Evito involucrarme en su trabajo

Trato de que obtengan lo que

deseo a cambio de su

cooperación

0.75

Siempre que lo crean necesario, pueden negociar conmigo qué obtendrán a cambio por su trabajo

Les hago saber que pueden lograr lo que quieren si trabajan conforme a lo pactado conmigo

Les doy lo que quieren a cambio de recibir su apoyo

Me aseguro de que exista un fuerte acuerdo entre lo que se espera que hagan y lo que pueden obtener de mí por su esfuerzo

Tienen plena confianza en mí

Cuento con su respeto

Se sienten orgullosos de trabajar conmigo

Confían en mi capacidad para superar cualquier obstáculo

Centro mi atención en los casos en los que no se consigue alcanzar las metas esperadas

Estoy dispuesto a instruirles o enseñarles siempre que lo necesiten

Me preocupo de formar a aquellos que lo necesiten

Les doy charlas para motivarlos

Trato de desarrollar nuevas formas para motivarlos

Potencio su motivación de éxito 
Análisis factorial confirmatorio

Como paso siguiente se verificó la estructura factorial obtenida por medio del análisis factorial confirmatorio. Los parámetros del modelo original propuesto fueron estimados siguiendo el criterio de Máxima Verosimilitud. Como entrada para el análisis se utilizó la matriz de correlaciones entre los ítems de la escala. En el Cuadro 2 se recoge la información proporcionada por seis de los índices de ajuste más utilizados (García-Cueto, Gallo \& Miranda, 1998): $\chi^{2} ; \chi^{2} /$ gl; GFI, índice de bondad de ajuste; AGFI, índice ajustado de bondad de ajuste; NFI, índice de ajuste normado; CFI, índice de ajuste comparado y RMSEA, error de aproximación cuadrático medio. Se verificó la estructura para un modelo de tres factores (liderazgo transformacional, transaccional y laissez faire) de forma conjunta y separada para los dos tipos de poblaciones estudiadas. Aunque, en los tres casos, la magnitud del estadístico $\chi^{2}$ es elevada y significativa, lo cual indica que los datos no se ajustan al modelo propuesto, se ha considerado de manera complementaria otros índices de ajuste que también se muestran en el Cuadro 2. Para las dos poblaciones combinadas el ajuste es aceptable; para población civil el ajuste, si bien es aceptable resulta algo pobre, y para población militar tenemos un ajuste casi óptimo. Para población general encontramos que las variables latentes liderazgo transformacional y laissez faire tenían correlaciones de 0.95 (validez convergente), posible razón del bajo ajuste del modelo de tres factores. Aparentemente la población general no discrimina entre ambas dimensiones del liderazgo (laissez faire y transaccional). Registramos correlaciones bajas entre la variable latente correspondiente al liderazgo transformacional y transaccional $(r=0.14)$ y correlaciones negativas con laissez faire $(r=-0.36)$. En cuanto a la población militar encontramos correlaciones negativas entre la variable latente correspondiente al liderazgo transformacional y laissez faire $(r=-0.39)$ y correlaciones bajas entre liderazgo transformacional y liderazgo transaccional $(r=0.15)$. En cuanto a la relación entre liderazgo transaccional y laissez faire encontramos correlaciones 
La evaluación de los estilos de liderazgo en población civil y militar argentina

moderadamente bajas ( $r=0.39$ ) Para esta población un modelo de tres factores tiene un ajuste muy bueno, con muy buena validez discriminante entre los tres factores propuestos.

\section{Cuadro 2}

Análisis factorial confirmatorio del MLQ-AVA. Índices de ajuste para un modelo de tres factores en población civil y militar

\begin{tabular}{lcccccccc}
\hline MLQ-AVA & \multicolumn{8}{c}{ Indices de ajuste } \\
& $\chi^{2}$ & $p$ & $\chi^{2 / g l}$ & $G F I$ & $A G F I$ & $N F I$ & $C F I$ & $R M S E A$ \\
\hline Población general & & & & & & & & \\
y militar & 150.50 & 0.01 & 2.95 & 0.93 & 0.89 & 0.87 & 0.90 & 0.07 \\
Población civil & 140.54 & 0.01 & 2.75 & 0.88 & 0.81 & 0.84 & 0.83 & 0.09 \\
Población militar & 78.21 & 0.01 & 1.53 & 0.93 & 0.89 & 0.84 & 0.93 & 0.06 \\
\hline
\end{tabular}

Consistencia interna

Una vez que se dispuso de la nueva versión del MLQ abreviada para población local fue preciso verificar si se trataba de un instrumento fiable y útil para evaluar los estilos de liderazgo según la teoría de Bass. Para estudiar la fiabilidad de cada una de las variables latentes se calculó el coeficiente alfa de Cronbach, obteniéndose fiabilidades bastante satisfactorias para cada una de las siete escalas. En el Cuadro 3 se citan dichos valores y se los compara con los obtenidos por el autor de la prueba con la muestra de estandarización original con propósitos ilustrativos (Bass \& Avolio, 1990). Como se puede apreciar, los índices de fiabilidad son buenos tanto para población civil como militar. El examen del cuadro permite asimismo verificar que no existen grandes discrepancias según el cuestionario se administre a población civil o militar. 


\section{Cuadro 3}

Coeficientes de fiabilidad MLQ-AVA (abreviado, versión argentina) - Población civil y militar

\begin{tabular}{lcccc}
\hline Dimensión & $\begin{array}{c}\text { Población } \\
\text { cadetes } \\
n=111\end{array}$ & $\begin{array}{c}\text { Población Población civil } \\
\text { oficiales } \\
n=61\end{array}$ & $\begin{array}{c}\text { Prgentina } \\
n=191\end{array}$ & $\begin{array}{c}\text { Norteamerican civil } \\
n=251\end{array}$ \\
\hline $\begin{array}{l}\text { Liderazgo transformacional } \\
\text { Carisma }\end{array}$ & & & & \\
Estimulación intelectual & 0.70 & 0.66 & 0.64 & 0.83 \\
Inspiración & 0.77 & 0.71 & 0.80 & 0.72 \\
Consideración individualizada & 0.63 & 0.60 & 0.61 & 0.60 \\
Liderazgo transaccional & & 0.60 & 0.60 & 0.71 \\
Recompensa contingente & 0.75 & 0.62 & 0.72 & 0.82 \\
Dirección por excepción & 0.76 & 0.73 & 0.70 & 0.62 \\
Laissez faire & 0.64 & 0.75 & 0.75 & 0.60 \\
\hline
\end{tabular}

\section{Validez de criterio}

Si el estilo de liderazgo predice en alguna medida la conducta concreta, se hipotetiza que aquellos cadetes con mejor rendimiento militar fueran los que puntúen más alto en las escalas de liderazgo transformacional y menos en la de laissez faire. Se analizó el rendimiento militar objetivo (evaluado por oficiales instructores) del grupo de cadetes y se calcularon las correlaciones con el MLQ. Se encontraron correlaciones negativas entre el rendimiento militar y estimulación intelectual $(r=-0,21, \mathrm{p}<0,05)$, dirección por excepción $(r=-0,23$, $p<0,05)$ y laissez-faire $(r=-0,21, \mathrm{p}<0,05)$. Es decir que los cadetes que exhibían peor performance militar eran aquellos que se autopercibían asimismo con menores características personales como para persuadir a los otros haciéndoles cambiar las expectativas, siendo más propensos a negociar con los seguidores en función de recompensas y castigos y con más conductas de laissez faire, tratando de intervenir lo menos posible. En la misma medida, según las propuestas de Bass, si el estilo de liderazgo predominante (percibido en el 
La evaluación de los estilos de liderazgo en población civil y militar argentina

superior) es transformacional y/o transaccional esto redundaría en una mayor satisfacción en el trabajo que si predominara el estilo laissez-faire. Se encontraron correlaciones positivas y significativas entre el liderazgo transformacional y la satisfacción en el trabajo ( $r=$ $0.47, p<0.01)$ para el grupo de líderes en población civil $(n=191)$. Asimismo, encontramos correlaciones moderadamente altas entre el liderazgo transaccional y la satisfacción laboral $(r=0.34, p<0.01)$. Entre el estilo laissez faire y la satisfacción no encontramos relación.

\section{Diferencias individuales entre población civil y militar}

Para analizar si existían diferencias por tipo de población se realizó un análisis multivariado de la varianza. Se introdujo como factor el tipo de población (civil, cadetes y oficiales). El análisis realizado arrojó diferencias estadísticamente significativas entre los tres vectores de medias $\left(\lambda=.78, \mathrm{~F}(3,345)=5.04, p<0.001, \eta^{2}=\right.$ 0.12) (Figura 1). Se llevó a cabo un análisis discriminante para establecer las subescalas que discriminaban más entre los tres grupos. Se obtuvo una función discriminante que era significativa $\left(\lambda=0.62, \chi^{2}\right.$ $=66, \mathrm{gl}=6, p<0.001$ ) y que explicaba un $35 \%$ de la varianza. Las escalas que discriminaban más eran liderazgo transaccional (Escala Total) (correlación con la función, $r=0.75$ ), inspiración intelectual (correlación con la función, $r=-0.61$ ) y consideración individualizada (correlación con la función, $r=-0.32$ ).

La población general civil se diferenciaba en mayor medida de la población militar en las características del liderazgo transaccional y la dimensión de inspiración intelectual en menor medida. Los líderes civiles son entonces más propensos a negociar intercambios con los seguidores y menos proclives a la persuasión para el cambio de creencias de los demás. En cambio en la población militar predominan menos las características del líder transaccional y se encuentran resaltadas la dimensión inspiración intelectual del liderazgo transformacional y la consideración individualizada. En la población militar 
para el ejercicio de la conducción importa más la motivación del subalterno y la resolución de situaciones a través del ejemplo personal. Esto se puede apreciar en mayor medida en la población de oficiales $y$ en un rango menor en la de cadetes.

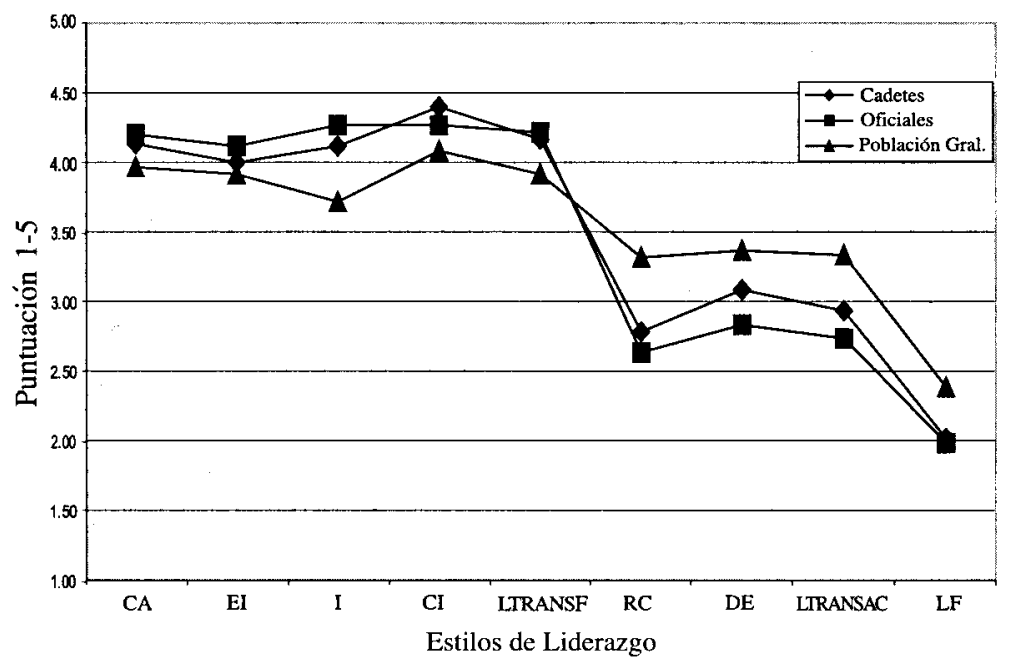

Figura 1. Estilos de liderazgo en población civil y militar.

$\mathrm{CA}=$ Carisma EI = Estimulación Intelectual $\mathrm{I}=$ Inspiración $; \mathrm{CI}=$ Consideración Individualizada; LTRANSF $=$ Liderazgo Transformacional; $\mathrm{RC}=$ Recompensa Contingente; $\mathrm{DE}=$ Dirección por Excepción; LTRANSAC = Liderazgo Transaccional; $\mathrm{LF}=$ Laissez Faire.

Autopercepción de liderazgo y percepción del superior

Para analizar si existían diferencias en la percepción del superior tanto de civiles y militares (cadetes y oficiales) se llevaron a cabo dos análisis discriminantes. El primero de ellos tenía como objetivo establecer la diferencia entre la percepción de liderazgo en el cuestionario autoadministrado y en el superior para la población de militares. Se obtuvo una función discriminante que era significativa $(\lambda=0.81$, 
$\left.\chi^{2}=110.26, g l=3, p<0.01\right)$ y que explicaba un $23 \%$ de la varianza. Las escalas que discriminaban más eran Carisma (correlación con la función, $r=-0.83$ ), inspiración intelectual (correlación con la función, $r=0.73$ ) y estimulación intelectual (correlación con la función, $r=0.86$ ). Los militares se autoperciben como más carismáticos, con mayores habilidades para inspirar y convencer a los demás que lo que ellos perciben en los superiores en los que estas características están menos presentes (ver Figura 2).

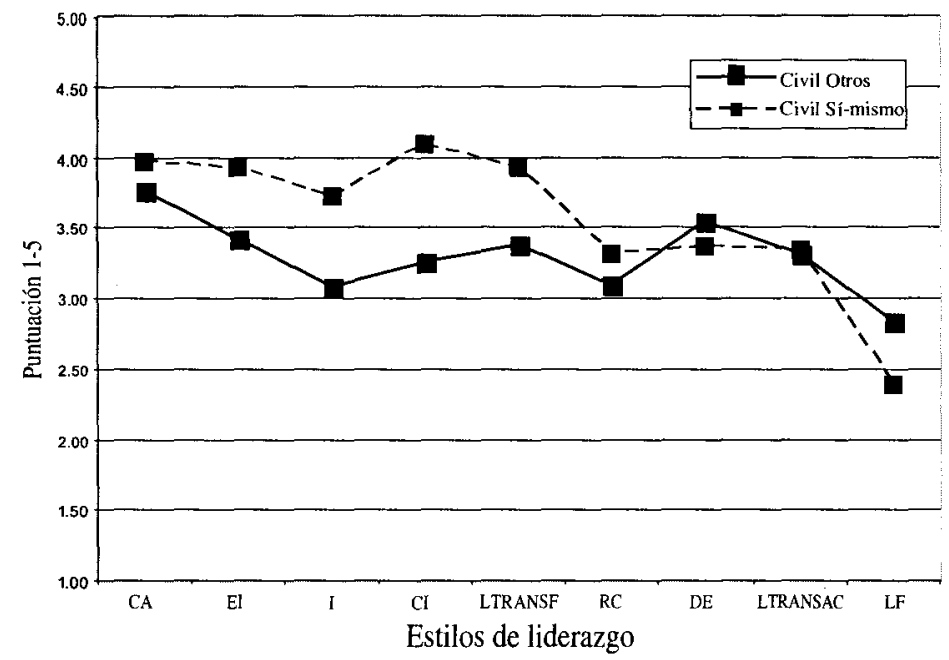

Figura 2. Autopercepción y percepción del estilo de liderazgo en el superior en cadetes.

$\mathrm{CA}=$ Carisma $; \mathrm{EI}=$ Estimulación Intelectual; $\mathrm{I}=$ Inspiración $; \mathrm{CI}=$ Consideración Individualizada; LTRANSF = Liderazgo Transformacional; $\mathrm{RC}=$ Recompensa Contingente; DE = Dirección por Excepción; LTRANSAC = Liderazgo Transaccional; LF $=$ Laissez Faire. 
El segundo análisis tenía como objetivo establecer la diferencia entre la percepción de liderazgo en el cuestionario autoadministrado y en el superior para la población de líderes civiles. Se obtuvo una función discriminante que era significativa $\left(\lambda=0.73, \chi^{2}=11616 \mathrm{gl}\right.$ $=4, p<0.01$ ) y que explicaba un $38 \%$ de la varianza. Las escalas que discriminaban más eran Consideración Individualizada (correlación con la función, $r=0.64$ ), Liderazgo Transformacional (correlación con la función, $r=0.53$ ) y Liderazgo Laissez Faire (correlación con la función, $r=-0.38$ ). Es decir, los civiles en algún cargo de dirección se percibían asimismo como teniendo mayor consideración por los subalternos y con más características de líderes transformacionales que lo que veían en su superior, al mismo tiempo se visualizaban con menores características de Laissez Faire (ver Figura 3).

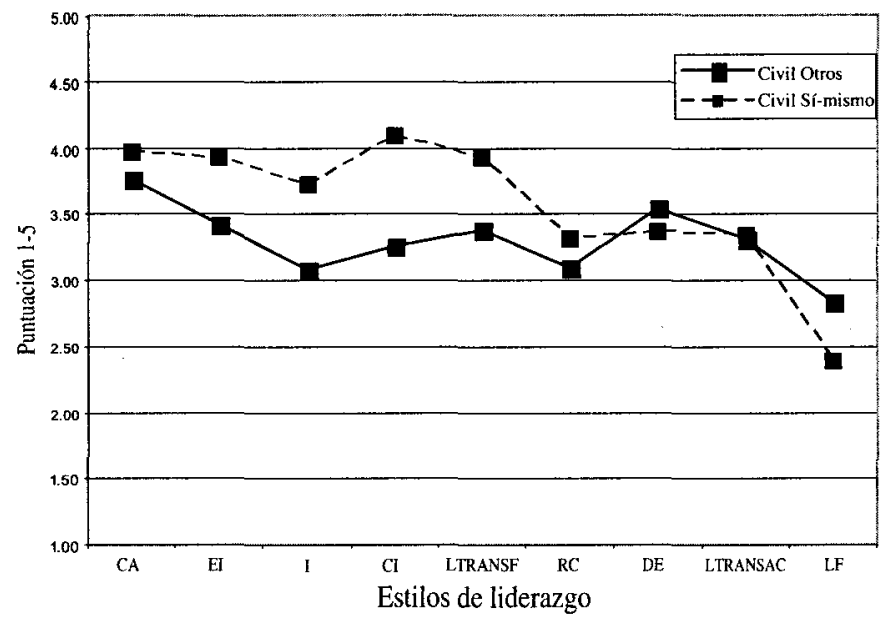

Figura 3. Autopercepción y percepción del estilo de liderazgo en el superior en población civil.

$\mathrm{CA}=$ Carisma; $\mathrm{EI}=$ Estimulación Intelectual; $\mathrm{I}=$ Inspiración; $\mathrm{CI}=$ Consideración Individualizada; $\mathrm{LTRANSF}=$ Liderazgo Transformacional; $\mathrm{RC}=$ Recompensa Contingente DE $=$ Dirección por Excepción $;$ LTRANSAC $=$ Liderazgo Transaccional; $\mathrm{LF}=$ Laissez Faire. 
La evaluación de los estilos de liderazgo en población civil y militar argentina

\section{Discusión}

El objetivo principal de este estudio era contar con una técnica válida y fiable para la evaluación de los estilos de liderazgo en población local. Los resultados de esta investigación señalan que la adaptación realizada del MLQ (versión argentina abreviada) tiene buenas propiedades psicométricas. Se obtuvo una solución de siete factores que se agrupaban en tres estilos de liderazgo. Estos datos fueron luego confirmados con técnicas de análisis factorial confirmatorio. Los análisis comentados indican un buen ajuste del modelo original propuesto por Bass (1990) para la población estudiada. El modelo exhibe un mejor ajuste para población militar que para población civil. En la población militar un modelo de liderazgo de tres factores (transformacional, transaccional y laissez faire) tiene un ajuste casi óptimo con muy buena validez discriminante. En población civil en cambio, los componentes relacionados con el liderazgo transaccional tienden a fundirse con los del laissez faire. Estos resultados son coincidentes con los obtenidos por Den Hartog, Van Muijen y Koopman (1997) con población holandesa civil que trabajaba en empresas públicas y privadas, en el cual los autores confirmaron un modelo de tres factores similar al nuestro. En la población civil el ajuste baja probablemente debido a las características de la muestra, siendo más heterogénea que la empleada para verificar el modelo correspondiente a población militar. En cuanto a las fiabilidades obtenidas se encuentran en valores entre .60 y .77 considerándose aceptables para este tipo de instrumento, resultando algo menores que las obtenidas en población norteamericana (Bass, 1990).

Asimismo confirmamos la utilidad de la prueba para predecir el rendimiento militar y la satisfacción en el trabajo. Un líder militar en formación (cadete) de características transformacionales es aquél que es evaluado con un rendimiento militar más alto por sus superiores. En la misma línea un estilo de liderazgo transformacional en un ámbito civil correlaciona en buena medida con buena satisfacción laboral y 
clima de trabajo. Estos resultados están en la línea de los obtenidos por Bass (1998) en diferentes tipos de poblaciones, en los cuales se verifica que el liderazgo transformacional es un importante predictor de efectividad en la tarea y de buen clima en la organización.

En cuanto a las diferencias entre los estilos de liderazgo en población civil y militar encontramos que los civiles en puestos de dirección tienden a exhibir características relacionadas con el intercambio y la negociación con los subalternos para la obtención de las metas organizacionales. En cambio, en los ámbitos militares las características más valoradas (autopercibidas) están más relacionadas con aspectos transformacionales ligados a la motivación del subalterno (consideración individualizada) y la forma en que los líderes transmiten el proyecto institucional a sus seguidores. Probablemente éstas sean las características necesarias para desarrollar la efectividad en un contexto más impredecible y cambiante, en donde el objetivo es mantener la cohesión frente a situaciones difusas y de cambio permanente (Bass et al., 2003).

Estas diferencias encontradas en los estilos de liderar en ambas poblaciones indican la necesidad de considerar el contexto en la evaluación del liderazgo. Es probable que los líderes pongan en práctica diferentes conductas y estrategias en función del contexto en el que les toca desempeñarse, por lo tanto un estilo de liderazgo transformacional puede ser más adaptativo en un contexto que en otro. Al igual que Antonakis et al. (2003) consideramos fundamental la consideración de los aspectos contextuales en la generación de teorías y en la evaluación de instrumentos de evaluación derivados.

Una diferencia interesante se encontró al comparar las características autopercibidas del líder y las percibidas en el superior. En términos generales los perfiles (tanto para militares cadetes como para civiles) seguían un patrón similar. Los cadetes con capacidad de mando se autopercibían con más atributos transformacionales que los 
superiores de los cuales dependían, básicamente carisma e inspiración de los demás. Encontramos una tendencia a percibir los atributos positivos incrementados en el formato de autopercepción y disminuido en la atribución que se hace del superior.

En población civil existía un mismo patrón pero los seguidores exhibían mayor discrepancia respecto de sus superiores. Estos se percibían a sí mismos con mayores características de líderes transformacionales predominando la consideración de los demás, en la misma línea se percibían con menores características de no líder (laissez faire).

Respecto del aspecto comentado no sabemos a ciencia cierta si esto es producto de un sesgo en la percepción o si se debe a características percibidas reales, siendo una limitación de la evaluación del liderazgo mediante métodos de encuesta.

En función de los resultados comentados resulta importante el estudio de las variables moderadoras en el ejercicio del liderazgo (House, Nages, Ruiz Quintanilla \& Dorfman, 1999). El liderazgo no es tan lineal como aparenta, sino que existen variables organizacionales que inciden sobre la concepción de líder esperado en función de las cuales no sólo un líder es identificado como tal, sino que su efectividad depende de los atributos que los seguidores le otorguen. La consideración de las normas culturales y su posible violación por parte del líder hace que una conducta muy efectiva y adaptativa en una situación resulte absolutamente contraindicada en otra distinta.

\section{Referencias}

Antonakis, J., Avolio, B. \& Sivasubramaniam, N. (2003). Context and leadership: An examination of the nine-factor Full Range Leadership Theory using the Multifactor Leadership Questionnaire. The Leadership Quarterly, 14(3), 261-295. 
Avolio, B. J. (1999). Full leadership development: Building the vital forces in the organization. Thousand Oaks, CA: Sage Publications. Avolio, B., Bass, B. \& Jung, D. (1995). MLQ Multifactor Leadership Questionnaire: Technical report. Redwgood City, CA: Mindgarden.

Bass, B. M. (1985). Leadership and performance beyond expectations. Nueva York: Free Press.

Bass, B. M. (1990). Handbook of leadership: A survey of theory and research. Nueva York: Free Press.

Bass, B. M. (1998). Transformational leadership: Industrial, military and educational impact. Mahwah, NJ: Lawrence Erlbaum.

Bass, B. M. \& Avolio, B. (1990). Manual for the Multifactor Leadership Questionnaire. Palo Alto, CA: Consulting Psychologists Press.

Bass, B. M. \& Avolio, B. (1993). Transformational leadership: A response to critiques. En M. Chemers \& R. Ayman (Eds.), Leadership theory and research: Perspectives and directions (pp. 49-80). San Diego, CA: Academic Press.

Bass, B. M. \& Avolio, B. (1994). Improving organizational effectiveness through transformational leadership. Thousand Oaks, CA: Sage Publications.

Bass, B. M. \& Avolio, B. (1997). Full range leadership development: Manual for the Multifactor Leadership Questionnaire. Palo Alto, CA: Mindgarden.

Bass, B. M., Avolio, B., Jung, D. \& Berson, I. (2003). Predicting unit performance by assessing transformational and transactional leadership. Journal of Applied Psychology, 88(2), 207-218.

Burns, J. M. (1978), Leadership. Nueva York: Harper \& Row.

Bycio, P., Hackett, R. \& Allen, J. (1995). Further assessment of Bass's (1985) conceptualization of transactional and transformational leadership. Journal of Applied Psychology, 80(4), 468-478.

Cantor, N. \& Mischel, W. (1979). Prototypes in person perception. En L. Berkovitz (Ed.), Advances in experimental social psychology. Nueva York: Academic Press. 
Carless, S. A. (1998). Assessing the discriminant validity of transformational leader behavior as measured by the MLQ. Journal of Occupational and Organizational Psychology, 71, 353-358.

Conger, J. \& Kanungo, R. (Eds.) (1988). Charismatic leadership: The elusive factor in organizational effectiveness. San Francisco: Jossey Bass.

Den Hartog, D., Van Muijen, J. \& Koopman, P. (1997). Transactional versus transformational leadership: An analysis of the MLQ. Journal of Occupational and Organizational Psychology, 70, 1934.

Fisher, B. \& Edwards, J. (1988). Considerating and initiating structure and their relationships with leader effectiveness. A metaanalysis. Proceedings of the Academy of Management, 201-205.

Gal, R. (1985). Commitment and obedience in the military: An Israeli case study. Armed Forces \& Society, 11, 553-564.

García-Cueto, E., Gallo Alvaro, P. \& Miranda, R. (1998). Bondad de ajuste en el análisis factorial confirmatorio. Psicothema, 10, 717-724.

House, R., Hanges, P., Ruiz Quintanilla, A. \& Dorfman, P. (1999). Emics and etics of culturally endorsed implicit leadership theories: Are attributes of charismatic/transformational leadership universally endorsed? The Leadership Quarterly, 10(2), 219-256. Hunt, J. G. (1991). Leadership. A new synthesis. Newbury Park, CA: Sage Publications.

Lowe, K., Kroeck, K. \& Sivasubramaniam, N. (1996). Effectiveness correlates of transformational and transactional leadership: A meta-analytic review. The Leadership Quarterly, 7, 385-425.

Morales, J. \& Molero, F. (1995). Leadership in two types of healthcare organization. En J. M. Peiró, F. Prieto, J. Meliá \& O. Luque (Eds.), Work and organizational psychology: European contributions of the nineties (pp. 209-221). Londres: Lawrence Erlbaum.

Paterson, C., Fuller, J., Kester, K. \& Stringer, D. (1995, abril). A meta-analytic examination of leadership style and selected compliance outcomes. Documento presentado en la décima conferen- 
cia anual de la Society of Industrial and Organizational Psychology, Orlando, FL, EE.UU.

Shamir, B., Zakay, E., Breinin, E. \& Popper, M. (1998). Correlates of charismatic leader behavior in military units: Subordinates' attitudes, unit characteristics and superiors' appraisals of leader performance. Academy of Management Journal, 41, 384-409.

Shamir, B., House, R. \& Arthur, M. (1993). The motivational effects of charismatic leadership. Organizational Science, 4, 577-594.

Stogdill, R., Good, O. \& Day, D. (1962). New leader behavior description subscales. Journal of Psychology, 54, 259-269.

Trice, H. \& Beyer, J. (1993). The cultures of work organizations. Englewood Cliffs, NJ: Prentice Hall.

Wofford, J., Godwin, V. \& Wittington, J. (1998). A field study of a cognitive approach to understanding transformational and transactional leadership. The Leadership Quarterly, 9(1), 55-84.

Yekeay, G. (2002). El liderazgo situacional. Military Review, 42-53. Yukl, G. (2002). Leadership in organizations. Englewood Cliffs, NJ:

Prentice Hall. 